Received: 2016.11 .05

Accepted: 2016.11 .28

Published: 2017.08.07

Authors' Contribution:
A Study Design
B Data Collection
C Statistical Analysis
D Data Interpretation
E Manuscript Preparation
F Literature Search
G Funds Collection

\section{Imaging Findings in a Rare Case of Leiomyomatosis Peritonealis Disseminata with Malignant Transformation}

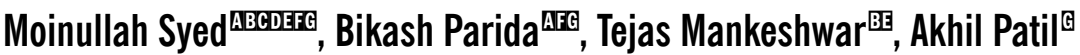 \\ Department of Radiodiagnosis, Byramjee Jeejeebhoy Government Medical College and Sassoon General Hospitals, Poona, \\ Maharashtra, India
}

Author's address: Moinullah Syed, Department of Radiodiagnosis, Byramjee Jeejeebhoy Government Medical College and Sassoon General Hospitals, Poona, Maharashtra, India, e-mail: moin.bmc@gmail.com

\begin{tabular}{c|l} 
Background: & $\begin{array}{l}\text { Summary } \\
\text { Leiomyomatosis peritonealis disseminata (LPD) is } \\
\text { of multiple leiomyomas in the abdominal and pe } \\
\text { LPD is an extremely rare occurrence. We hereby } \\
\text { subsequently diagnosed with leiomyomatosis perito } \\
\text { showing malignant transformation. }\end{array}$ \\
Case Report: & $\begin{array}{l}\text { A forty-year-old female with a history of hysterect } \\
\text { completely evaluated with ultrasound (US), comp } \\
\text { imaging (MRI) and was subsequently diagnosed with } \\
\text { imaging findings of leiomyomatosis peritonealis d } \\
\text { by a histopathologic examination of the excision biops } \\
\text { The imaging findings were found to be specific for } \\
\text { malignant transformation. }\end{array}$ \\
MeSH Keywords: & $\begin{array}{l}\text { Hysterectomy • Leiomyomatosis • Peritoneum } \\
\text { PDF file: }\end{array}$ \\
http://www.polradiol.com/abstract/index/idArt/902242
\end{tabular}

\section{Background}

Leiomyomatosis peritonealis disseminata is a rare disease of smooth muscle cells characterized by formation of multiple nodular masses in the peritoneum, seen almost exclusively in females, particularly of child-bearing potential and middle-aged. Malignant degeneration is an unusual complication of leiomyomatosis peritonealis disseminata, with only three cases reported in the literature so far. This entity can be easily misdiagnosed as a metastasis of an unknown primary without typical history leading to false positive diagnoses and inadvertent surgeries. In this article, we report a case of a female patient with a remote history of hysterectomy for uterine fibroid who was subsequently diagnosed with LPD with a malignant transformation.

\section{Case Report}

A forty-year-old woman presented to our department with vague abdominal pain for 7 months. She had a prior history of hysterectomy for multiple uterine fibroids three years earlier and was a regular oral contraceptive pill user. Plain abdominal radiograph of the patient was normal. On ultrasound, multiple, well-defined, lobulated, solid, hypoechoic mass lesions were noted in the abdomen and pelvis (Figure 1A, 1B). On a color Doppler study, few of these lesions showed significant internal vascularity with a low-resistance arterial waveform (Figure 2). The patient was then evaluated by plain and contrast-enhanced computed tomography which revealed multiple lobulated, isoto hypodense lesions in the peritoneum, bilateral adnexa, recto-uterine pouch, pre-vesical space and left rectus abdominis muscle (Figure 3). Most of these lesions showed moderate to intense enhancement on postcontrast images (Figure 4). Few of these enhancing lesions showed nonenhancing areas within, suggestive of necrosis. The patient was subsequently evaluated with MRI. The same lesions appeared isointense to skeletal muscles on TlW images (Figure 5), isointense to moderately hyperintense on T2W images (Figure 6) and moderately hyperintense on short tau inversion recovery (STIR) images (Figure 7). Central areas 


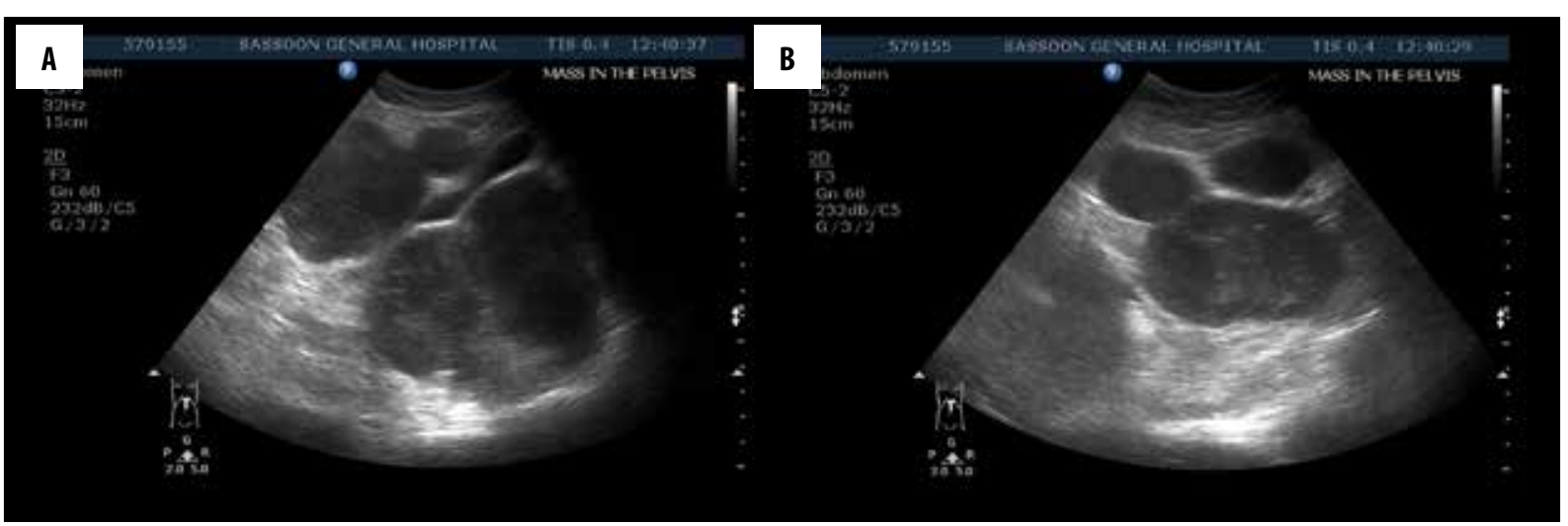

Figure 1. (A, B) Ultrasound images show multiple, well-defined, homogenous hypoechoic lesions in the pelvis and peritoneum.

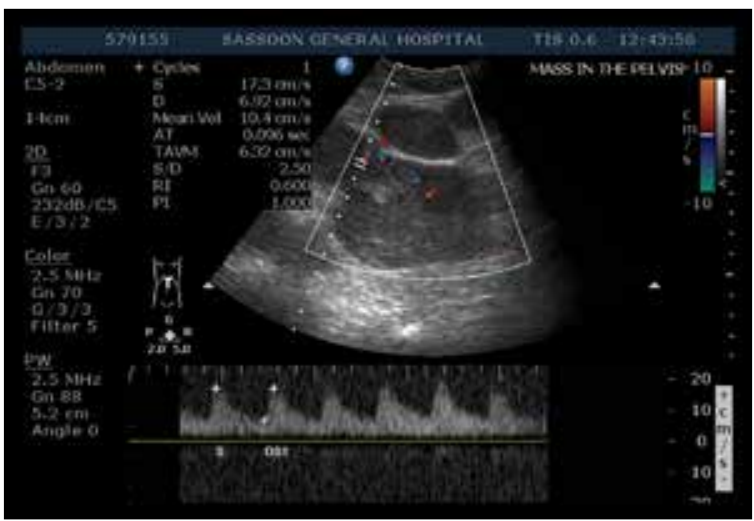

Figure 2. Color and spectral Doppler of one of the lesions shows a low-resistance arterial waveform.

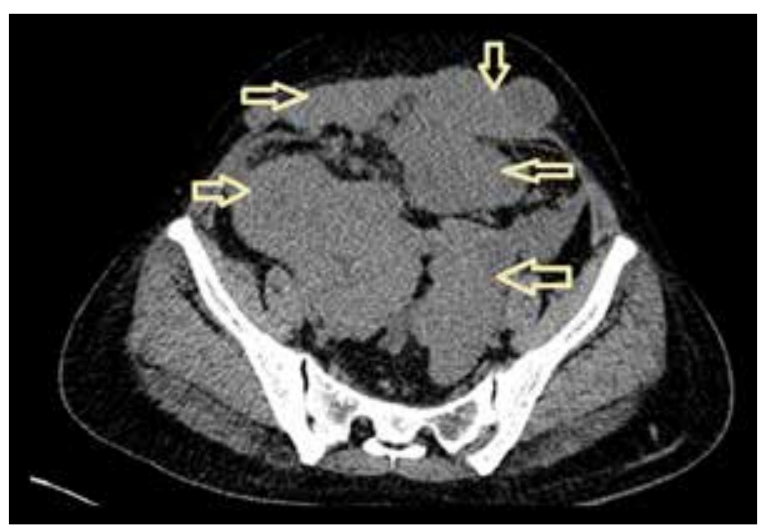

Figure 3. Axial plain CT image of the pelvis shows multiple, welldefined, isodense lesions as indicated by arrows.

of blooming were seen in few of these lesions on gradient echo (GRE) images, suggestive of hemorrhage (Figure 8). No restricted diffusion was noted in these lesions on diffusion weighted images (DWI). On postcontrast TlW images, intense enhancement of these lesions was seen with few non-enhancing areas within due to necrosis (Figure 9). The patient underwent exploratory laparotomy with bilateral oophorectomy and most of the lesions were resected from the peritoneum and pelvis. On gross appearance, the lesions appeared pale, lobulated and firm in consistency (Figure 10). On histopathological examination, the specimens consisted of spindle-shaped smooth muscle cells with intervening extracellular matrix and fibroblasts, consistent

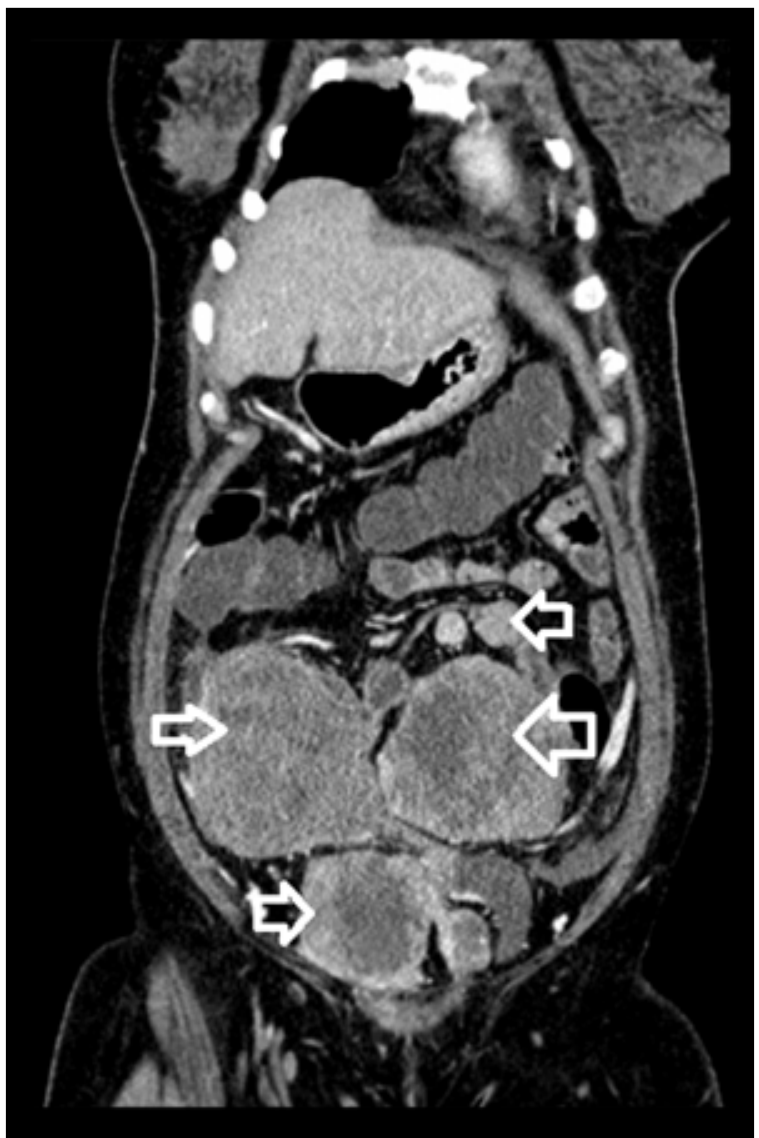

Figure 4. Coronal, reformatted, postcontrast CT image shows heterogeneous enhancement of the above-seen lesions with central non-enhancing areas suggestive of necrosis.

with leiomyoma (Figures 11, 12). Two of the specimens showed cellular atypia, pleomorphism and increased mitotic activity suggestive of a malignant transformation (Figure 13).

\section{Discussion}

Leiomyomatosis peritonealis disseminata is a rare disease of smooth muscle cells and is characterized by formation of multiple nodular masses in the abdominopelvic peritoneum, in the submesothelial tissue. Although the disease was first described as early as in 1952, the term was first 


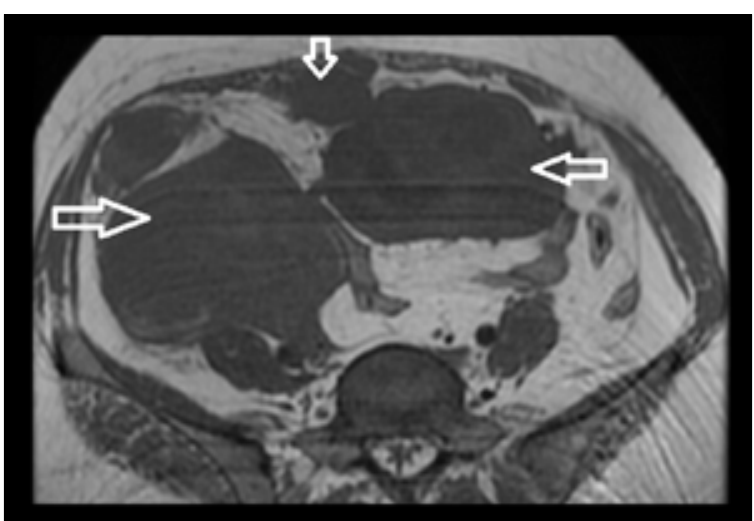

Figure 5. Axial T1W MR image of the pelvis shows multiple, welldefined, iso- to hypointense lesions as indicated by arrows.

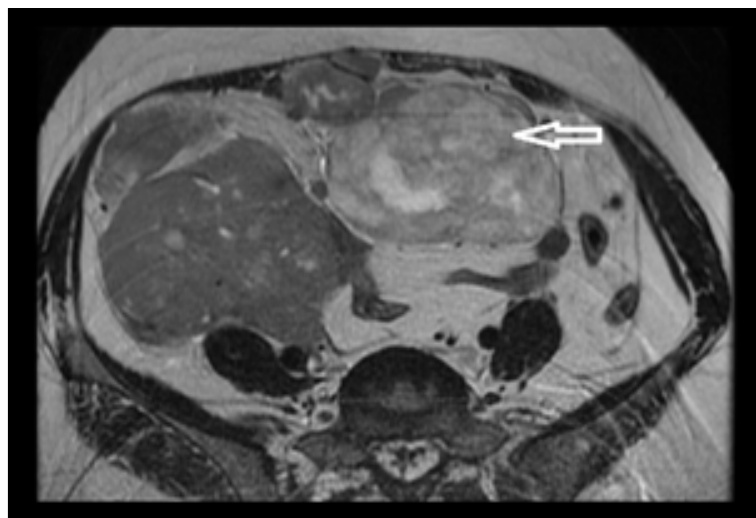

Figure 6. Axial T2W MR image at same level as in Figure 5 shows heterogeneous, predominantly hyperintense signal within the mass with cystic areas within. The lesion with the higher signal intensity is indicated by the arrow.

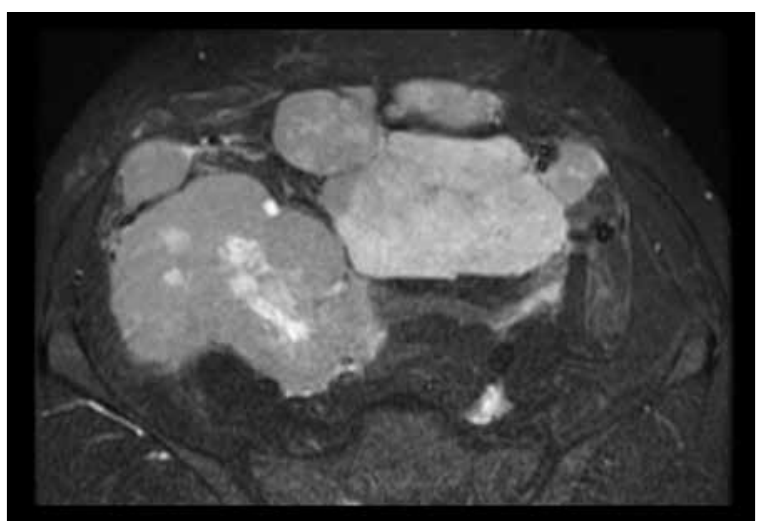

Figure 7. Axial, STIR MR image at same level as in Figure 9 shows hyperintense signal of the same lesions.

coined and explained in detail by Taubert in 1965 [1]. The pathogenesis of this condition has been attributed to exposure to increased levels of either endogenous or exogenous estrogens. An association has been noted with oral contraceptive pills and estrogen secreting tumors [2]. A lower sensitivity threshold of the smooth muscle cells to estrogens and subsequent metaplasia have also been suspected as a causative factor [3]. Most of the cases are usually asymptomatic and are associated with uterine leiomyomas. Laparoscopic myomectomy has also been hypothesized to

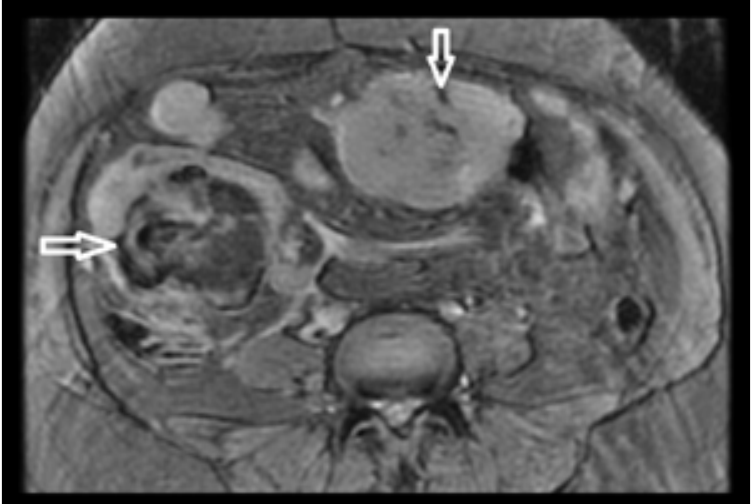

Figure 8. Axial, GRE MR image shows central areas of blooming within the masses as indicated by the arrows.

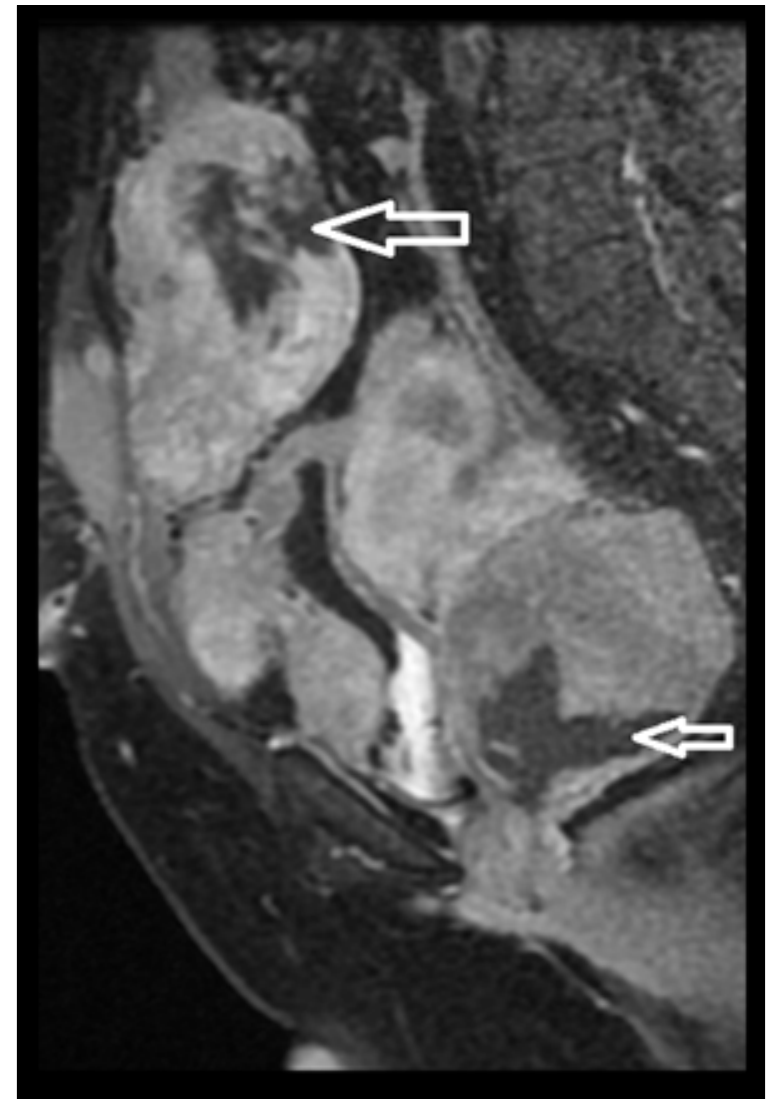

Figure 9. Sagittal, oblique, postcontrast, fat-sat MR image shows intense enhancement of the lesions with central areas of non-enhancement suggestive of necrosis (arrows).

cause LPD by a spread of smooth muscle cells along the laparoscopic tract [4]. Malignant transformation of LPD is an extremely rare complication of the disease with only three cases reported in the literature so far [5]. On ultrasound, the lesions are seen as multiple, lobulated, hypoechoic masses of variable sizes, scattered in the abdominopelvic peritoneum and omentum. Malignant lesions show internal vascularity with low resistance, as seen in the above-mentioned patient. Areas of hemorrhage and necrosis can also be noted in malignant lesions. Computed tomography shows these lesions as lobulated iso- to hypodense lesions with homogenous to heterogeneous 


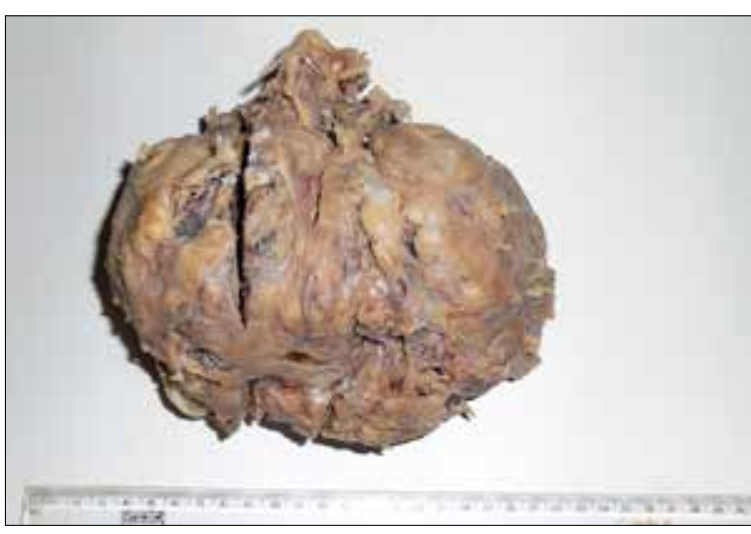

Figure 10. Gross surgical specimen of one of the lesions shows a pale, lobulated, solid mass.

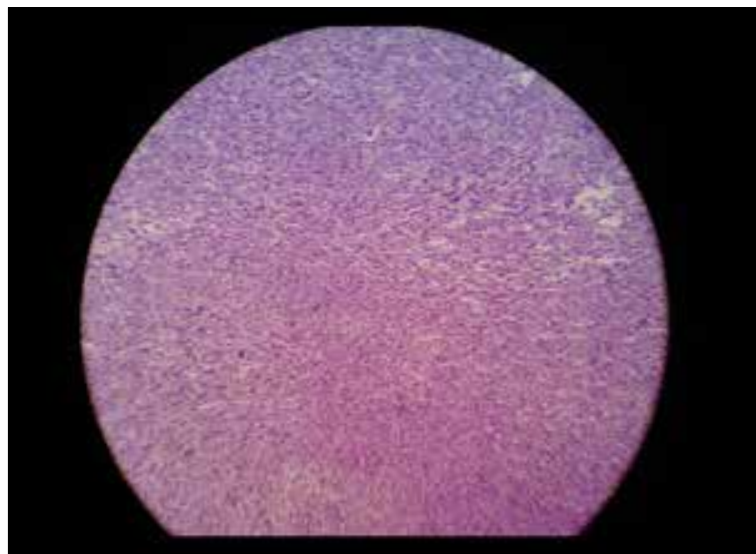

Figure 11. Low power microscopic histopathologic section of a lesion shows abundant spindle-shaped cells in bundles.

enhancement. Areas of necrosis and hemorrhage point towards a suspicion of malignancy. MRI is the imaging modality of choice for LPD due to its excellent soft tissue characterization. Typically, the lesions appear isointense to skeletal and smooth muscles on TlW and T2W images, with intense enhancement on postcontrast TlW images. Areas of increased signal intensity on T2W and STIR images with blooming on GRE images suggest necrosis and hemorrhage, respectively, which also points towards a malignant transformation. Diffusion weighted images also provide useful information, as malignant leiomyomas could show restricted diffusion with decreased apparent diffusion coefficient (ADC) values compared to their benign counterparts [6]. However, in our case, none of the lesions showed restricted diffusion on DWI.

Definitive diagnosis of LPD with a malignant transformation is made by a histopathological examination of a tissue sample, which usually reveals spindle-shaped cells, fibroblasts with cellular atypia and nuclear pleomorphism, intranuclear vacuoles and increased mitoses. On immunohistochemistry, the cells are positive for $\alpha$-smooth muscle actin and vimentin [7].

The main differential diagnosis of LPD is peritoneal carcinomatosis due to unknown primary, if patients present

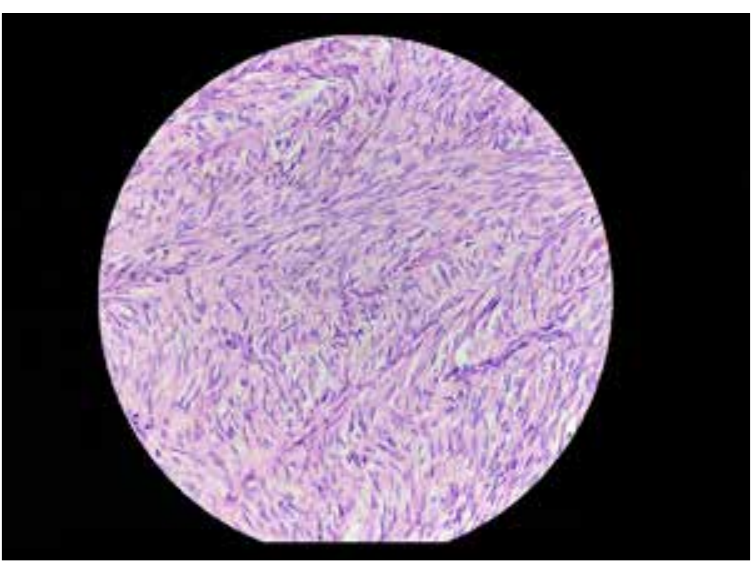

Figure 12. High power microscopic histopathologic section of the same slide shows spindle-shaped cells in bundles with few fibroblasts without cellular atypia or pleomorphism.

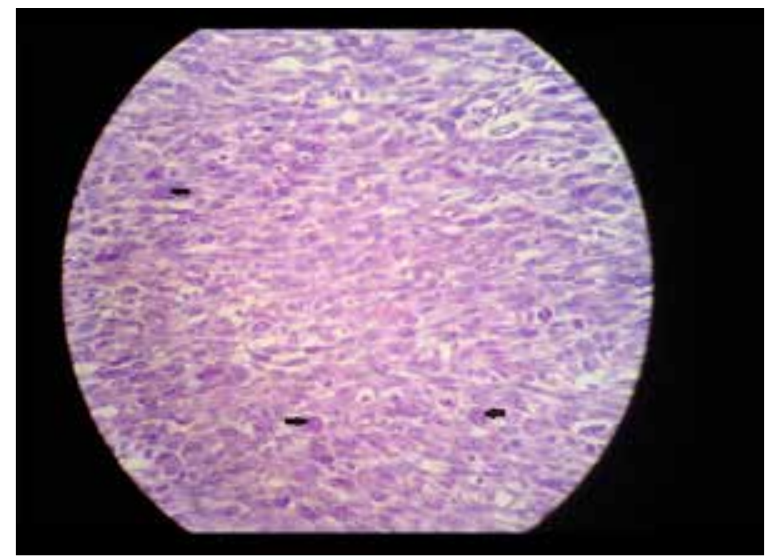

Figure 13. High power microscopic histopathologic section of another lesion shows cellular atypia with nuclear pleomorphism and increased mitotic figures (arrows).

with anorexia and cachexia [8]. Ascites, multiple bony and hepatic metastases point towards a diagnosis of carcinomatosis. Benign metastasizing leiomyoma is another major differential diagnosis in which multiple differentiated leiomyomas are seen in organs distant from the uterus. However, lesions are most commonly seen in the lungs. Concurrent presence of LPD and leiomyosarcoma can also be considered as a remote possibility [9].

\section{Conclusions}

Based on imaging findings and a pathologic correlation, a final diagnosis of leiomyomatosis peritonealis disseminata with a malignant transformation was made.

\section{Acknowledgement}

We would like to express our heartfelt gratitude to Dr. Sayali Deshmukh from Department of Pathology, B.J.Government Medical College, Poona and Dr. Hadiya Naureen from Department of Pathology, Bapuji Hospital for their selfless help in this article. 


\section{References:}

1. Taubert HD, Wissner SE, Haskins AL: Leiomyomatosis peritonealis disseminata. Obstet Gynecol, 1965; 25: 561-74

2. Drake A, Dhundee J, Buckley CH, Woolas R: Disseminated leiomyomatosis peritonealis in association with oestrogen secreting ovarian fibrothecoma. Br J Obstet Gynaecol, 2001; 108: 661-64

3. Travassoli FA, Norris HJ: Peritoneal leiomyomatosis (leiomyomatosis peritonealis disseminata): A clinicopathologic study of 20 cases with ultrastructural observations. Int J Gynecol Pathol, 1982; 1: 59-74

4. Kumar S, Sharma JB, Verma D et al: Disseminated peritoneal leiomyomatosis: an unusual complication of laparoscopic myomectomy. Arch Gynecol Obstet, 2008; 278(1): 93-95

5. Bekkers RL, Willemsen WN, Schijf CP et al: Leiomyomatosis peritonealis disseminata: Does malignant transformation occur? A literature review. Gynecol Oncol, 1999; 75(1): 158-63
6. Sato K, Yuasa N, Fujita M, Fukushima Y: Clinical application of diffusion-weighted imaging for preoperative differentiation between uterine leiomyoma and leiomyosarcoma. Am J Obstet Gynecol, 2014; 210(4): 368.el-8

7. Yamaguchi T, Imamura Y, Yamamoto T, Fukuda M: Leiomyomatosis peritonealis disseminata with malignant change in a man. Pathol Int, 2003; 53: 179-85

8. Fasih N, Prasad Shanbhogue AK, Macdonald DB et al: Leiomyomas beyond the uterus: Unusual locations, rare manifestations. Radiographics, 2008; 28: 1931-48

9. Tun AM, Tun NM, Zin Thein K: A rare concurrence of leiomyomatosis peritonealis disseminata, leiomyosarcoma of the pelvis and leiomyomatous nodule of the liver. Case Rep Oncol Med, 2016; 2016: 3025432 